\title{
Tectonic 1 accelerates gastric cancer cell proliferation and cell cycle progression in vitro
}

\author{
XINBAO WANG ${ }^{1}$, QIMING YU ${ }^{1}$, YINGLI ZHANG ${ }^{2}$, ZHIQIANG LING ${ }^{3}$ and PENGFEI YU ${ }^{1}$ \\ Departments of ${ }^{1}$ Abdominal Tumor Surgery, ${ }^{2}$ Clinic and ${ }^{3}$ Cancer Research Institute, Zhejiang Cancer Hospital, \\ Zhejiang Cancer Center, Banshanqiao, Hangzhou 310022, P.R. China
}

Received May 26, 2014; Accepted February 3, 2015

DOI: $10.3892 / \mathrm{mmr} .2015 .4177$

\begin{abstract}
Hedgehog (Hh) pathway is important in development and cancer. Hh signaling is constitutively active in gastric cancer. Recently, tectonic 1 (TCTN1) was identified as one regulator of the Hh pathway. In the present study, the biological role of TCTN1 was examined in gastric cancer via an RNA interference lentivirus system. The constructed lentivirus efficiently suppressed TCTN1 expression in three gastric cancer cell lines. The proliferation of gastric cancer cells was significantly inhibited in TCTN1 knockdown cells, as determined by 3-(4,5-dimethylthiazol-2-yl)-2,5-diphenyltetrazolium bromide and colony formation assays. Furthermore, in order to determine the underlying mechanism, the cell cycle progression of MGC80-3 cells was analyzed by flow cytometry. Knockdown of TCTN1 led to cell cycle arrest at the $\mathrm{G}_{2} / \mathrm{M}$ phase, which contributed to inhibition of growth. In conclusion, the results demonstrated that TCTN1 was essential in the growth of gastric cancer cells in vitro, suggesting TCTN1 as a potential target candidate for the treatment of gastric cancer.
\end{abstract}

\section{Introduction}

Gastric cancer is one of the leading causes of cancer-related mortality worldwide $(1,2)$. It remains difficult to cure, primarily because the majority of patients present with advanced-stage disease. The pathogenesis of gastric cancer is complex and relates to multiple factors. Dysregulation of intracellular signaling pathways represents a common pathogenic mechanism and may be amenable to drug targeting.

Hedgehog ( $\mathrm{Hh})$ signaling is critical during embryonic development. It is involved in the patterning of the

Correspondence to: Professor Xinbao Wang, Department of Abdominal Tumor Surgery, Zhejiang Cancer Hospital, Zhejiang Cancer Center, 38 Guangji Road, Banshanqiao, Hangzhou 310022, P.R. China

E-mail: xinbaodr@163.com

Key words: tectonic 1, gastric cancer, lentivirus, proliferation, cell cycle neural tube, lung, skin, axial skeleton and gastrointestinal tract (3-5). In multiple adult tissues, Hh signaling remains active and contributes to differentiation, proliferation and maintenance $(6,7)$. Deregulation of the Hh pathway is associated with congenital defects and cancer. Specifically, Hh signaling is constitutively active in a number of types of cancer, including gastric cancer (8), pancreatic cancer (9), prostate cancer (10), breast cancer (11), basal cell carcinoma (12) and small cell lung cancer (13). Elevated expression of Hh target genes patched gene 1 (Ptch1) or Gli1 occurs in $>2 / 3$ of primary gastric cancers (1). Sonic hedgehog (Shh) signaling promotes motility and invasiveness of gastric cancer cells through cross-talk with the transforming growth factor- $\beta$ pathway $(14,15)$. Recent studies have observed the presence of Smoothened (Smo) and/or Ptch1 mutations at a low frequency in different histological subtypes of gastric cancer (16). Rab23, another mediator of the Hh pathway, is also associated with gastric cancer $(17,18)$. These studies suggest that the molecules involved in the Hh pathway may be potential candidates for the treatment of gastric cancer (19).

Tectonic1 (TCTN1), which is a member of the tectonic family, has been identified as a regulator of the $\mathrm{Hh}$ pathway (20). TCTN1 functions in Hh signal transduction to fully activate the pathway in the presence of high $\mathrm{Hh}$ levels, and to repress the pathway by modulating Hh signal transduction downstream of Smo and Rab23 in the absence of Hh signals $(20,21)$. The expression pattern of TCTN1 resembles certain proteins in the Hh pathway. TCTN1-null mice showed similar phenotypes to those with Shh mutations. Recently, TCTN1 was reported to form a complex with multiple ciliopathy proteins associated with Meckel and Joubert syndromes $(21,22)$. Abolition of TCTN1 leads to tissue-specific defects in ciliogenesis and ciliary membrane composition (21). However, there have been no studies regarding the function of TCTN1 in human cancer thus far.

Accumulating evidence has shown that there is a close association between abnormal cell proliferation and cancer. In order to investigate the role of TCTN1 in gastric cancer, the present study investigated the biological role of TCTN1 in cell growth via an RNA interference lentivirus system in three gastric cancer cell lines. Cell proliferation, colony formation and cell cycle progression were investigated in gastric cancer cells following TCTN1 knockdown. 


\section{Materials and methods}

Cell culture. SGC-7901, MGC80-3 and AGS human gastric cancer cell lines and the 293T human embryonic kidney cell line (HEK293T) were obtained from the Cell Bank of the Chinese Academy of Sciences (Shanghai, China). SGC-7901 cells were maintained in RPMI-1640 (Hyclone, Logan, UT, USA) supplemented with $10 \%$ fetal bovine serum (FBS; Biowest, Kansas City, MO, USA). AGS cells were cultured in F-12 (Sigma-Aldrich, St. Louis, CA, USA) with 10\% FBS. MGC80-3 and HEK293T cells were cultured in Dulbecco's modified Eagle's medium (Hyclone) with $10 \%$ FBS. Cells were maintained at $37^{\circ} \mathrm{C}$ in a humidified atmosphere of $5 \% \mathrm{CO}_{2}$.

Lentiviral vector construction. The short hairpin RNA (shRNA) sequence (5'-GCTCAGATGCATCAGTTCCTTC TCG AGAAGGAACTGATGCATCTGAGCTTTTTT-3') was designed to target the human TCTN1 gene (NM_001082537.2). The control shRNA sequence was 5'-TTCTCCGAACGT GTCACG TCTCGAGACGTGACACGTTCGGAGAA-3'. The shRNA oligos were annealed, ligated and inserted into NheI/PacI double digested pFH-L vectors (Shanghai Hollybio, Shanghai, China). The generated plasmids were termed pFH-L-shTCTN1 or pFH-L-shCon.

Lentiviral packaging and cell infection. The reconstructed plasmids were then transfected into HEK293T cells using Lipofectamine 2000 (Invitrogen Life Technologies, Carlsbad, CA, USA) together with the helper plasmids pVSVG-I and pCMV-R8.92 (Shanghai Hollybio). Supernatants containing either the lentivirus expressing the TCTN1 shRNA (Lv-shTCTN1) or the control shRNA (Lv-shCon) were harvested $72 \mathrm{~h}$ after transfection. The lentiviruses were purified using ultracentrifugation $\left(4^{\circ} \mathrm{C}, 100,000 \mathrm{xg}, 2 \mathrm{~h}\right)$. As the lentivirus carried a green fluorescence protein (GFP) reporter, the viral titer was determined by counting GFP-expressing cells under a fluorescence microscope (Olympus BX50; Olympus Corporation, Tokyo, Japan) as described in previous studies $(23,24)$.

For cell infection, SGC-7901 (5x104 cells/well), MGC80-3 $\left(5 \times 10^{4}\right.$ cells/well $)$ and AGS $\left(8 \times 10^{4}\right.$ cells/well) were seeded into 6-well plates and incubated with recombinant lentivirus at a multiplicity of infection of 30,6 or 20 , respectively. At $72 \mathrm{~h}$ post infection, cells were observed under a fluorescence microscope. The infection efficiency was calculated as the number of GFP positive cells versus the total number of cells.

Reverse transcription-quantitative polymerase chain reaction $(R T-q P C R)$. Five days after lentivirus infection, SGC-7901, MGC80-3 and AGS cells were collected and extracted for total RNA using TRIzol reagent (Invitrogen Life Technologies) according to manufacturer's instructions. cDNA that was adjusted to a final concentration of $30 \mathrm{ng} / \mu \mathrm{l}$ was synthesized by using M-MLV reverse transcriptase (Promega Corporation, Madison, CA, USA). RT-qPCR was then performed to analyze the expression level of TCTN1 using actin as an endogenous control. Primers were designed as follows: Forward: 5'-CCTTTGCGTGAATGTTGTTC-3' and reverse: 5'-AGAGGGACTGGCTGGGTATT-3' for TCTN1; and forward: 5'-GTGGACATCCGCAAAGAC-3' and reverse:
5'-AAAGGGTGTAACGCAACTA-3' for actin. The RT-qPCR reaction system (Takara, Tokyo, Japan) was set up as follows: $10 \mu \mathrm{l}$ of $2 \mathrm{X}$ SYBR premix ex taq, $0.8 \mu \mathrm{l}$ of $2.5 \mu \mathrm{M}$ forward and

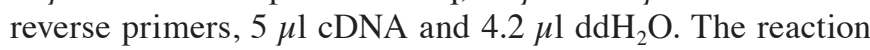
conditions were an initial denaturation step at $95^{\circ} \mathrm{C}$ for $1 \mathrm{~min}$ and 40 cycles of denaturation at $95^{\circ} \mathrm{C}$ for $5 \mathrm{sec}$ followed by annealing extension at $60^{\circ} \mathrm{C}$ for $20 \mathrm{sec}$. The absorbance values were obtained at the extension stage and expression levels were calculated using $2^{-\Delta \Delta C t}$ methods. Results were presented as Ct values, which were defined as the threshold PCR cycle number at which an amplified product is first detected. The average $\mathrm{Ct}$ was calculated for TCTN1 and actin, and $\Delta \mathrm{Ct}$ was determined as the ratio of the mean of the triplicate $\mathrm{Ct}$ values for TCTN1 to the mean of the triplicate $\mathrm{Ct}$ values for actin. The experiment was repeated at least three times on a BioRad Connet Real-Time PCR platform (Bio-Rad CFX96; Bio-Rad Laboratories, Inc., Hercules, CA, USA).

3-(4, 5-Dimethylthiazol-2-yl)-2, 5-diphenyltetrazolium bromide (MTT) assay. Three days after lentivirus infection, SGC-7901 (2.5x10 cells/well), MGC80-3 ( $2 \times 10^{3}$ cells/well) and AGS $\left(2 \times 10^{3}\right.$ cells/well $)$ were reseeded in 96 -well plates and cultured at $37^{\circ} \mathrm{C}$. The number of viable cells was measured at daily intervals (days 1, 2, 3,4 and 5). At each time point, $10 \mu \mathrm{l}$ of $5 \mathrm{mg} / \mathrm{ml}$ MTT was added to the cells. After incubation for $3 \mathrm{~h}$, acidic isopropanol (10\% SDS, 5\% isopropanol and $0.01 \mathrm{~mol} / \mathrm{l} \mathrm{HCl}$ ) was added. The absorbance value of each well was recorded at a wavelength of $595 \mathrm{~nm}$ (Epoch; BioTek, Winooski, VT, USA). Each experiment was repeated at least three times.

Colony formation assay. Three days after lentivirus infection, MGC80-3 cells were reseeded into 6-well plates at a density of 400 cells/well. The medium was changed at three-day intervals. After 7 days of culture at $37^{\circ} \mathrm{C}$, cells were fixed with $4 \%$ paraformaldehyde and crystal violet staining was performed. The colony number in each well was counted under a microscope (Olympus CH-2; Olympus Corporation). Image analysis was conducted using Image-Pro ${ }^{\circledR}$ Plus Version 6.0 (Media Cybernetics, MD, USA). Each experiment was repeated at least three times.

Flow cytometric analysis. Three days after lentivirus infection, MGC80-3 cells were reseeded in 6-cm dishes at a density of $2 \times 10^{5}$ cells/dish. When the confluence of MGC80-3 cells was $\sim 70 \%$, cells were collected and fixed with $70 \%$ cold ethanol overnight at $4^{\circ} \mathrm{C}$. Propidium iodide (PI; (C1052, Beyotime Institute of Biotechnology, Haimen, China) was then added to stain the nuclei following the manufacturer's instructions. The fluorescence of PI was measured using flow cytometry with Cell Lab Quanta Beckman Coulter (Gallios, Beckman Coulter, San Jose, CA, USA). Each experiment was repeated at least three times.

Statistical analysis. All data were presented as the mean \pm standard error of three independent experiments. SPSS 13.0 (SPSS, Inc., Chicago, IL, USA) was used for all statistical analyses. Comparison of data was analyzed using Student's t-test. $\mathrm{P}<0.05$ were considered to indicate a statistically significant difference. 
A

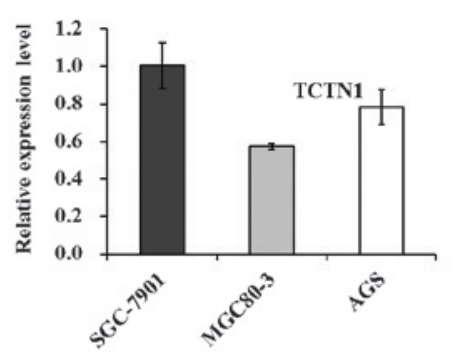

C

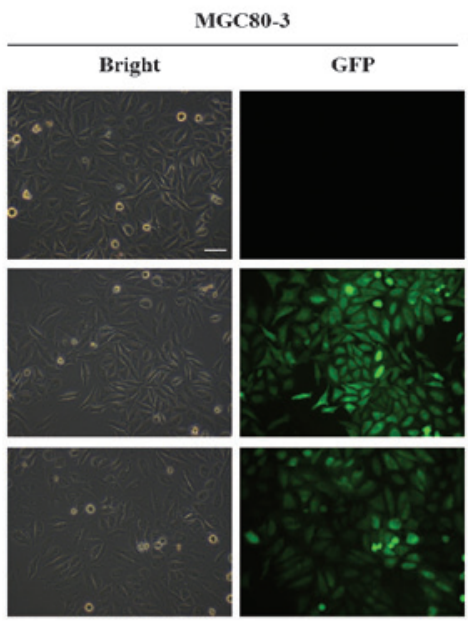

B
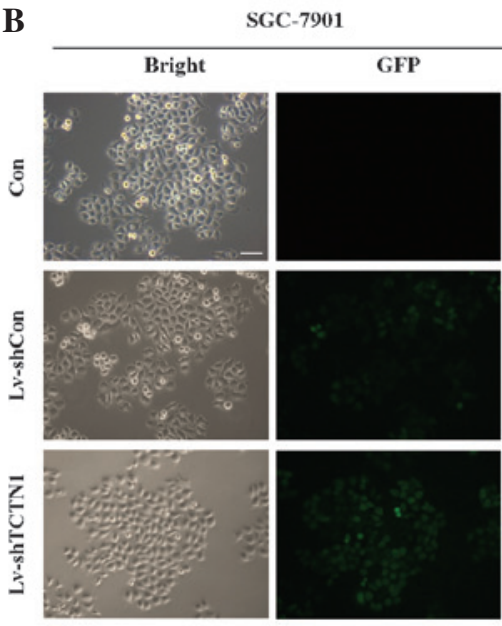

D

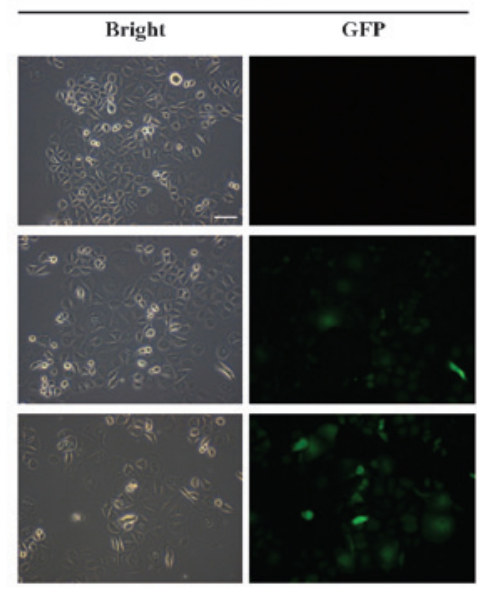

$\mathbf{E}$

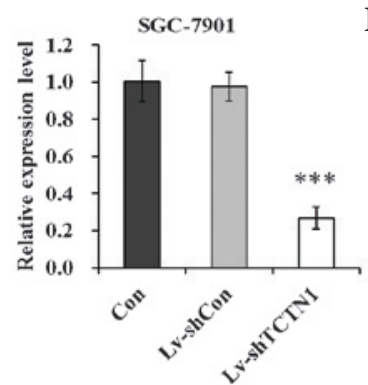

$\mathbf{F}$
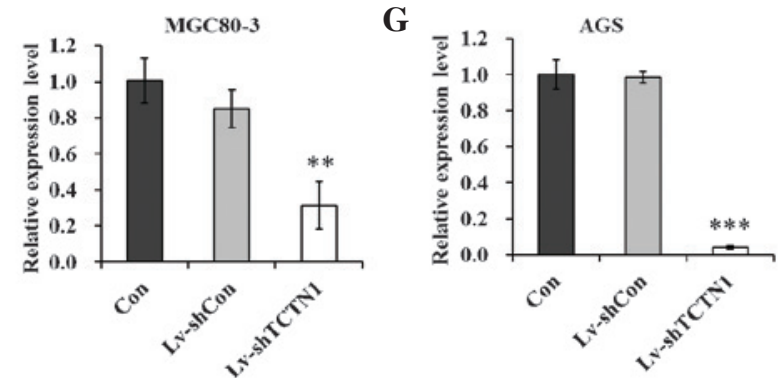

Figure 1. TCTN1 expression is efficiently suppressed in gastric cancer cells by Lv-shTCTN1. (A) RT-qPCR analysis of TCTH1 expression in three human gastric cancer cell lines. Representative images of lentivirus infection in (B) SGC-7901, (C) MGC80-3 and (D) AGS cells under a fluorescence microscope (scale bar, $50 \mu \mathrm{m}$ ). RT-qPCR analysis of TCTN1 knockdown efficiency in (E) SGC-7901, (F) MGC80-3 and (G) AGS cells. Actin was used as a control. *"P<0.01 and ${ }_{* * * *} \mathrm{P}<0.001$, compared with Lv-shCon. RT-qPCR, reverse transcription-quantitative polymerase chain reaction; TCTN1, tectonic 1 .

A

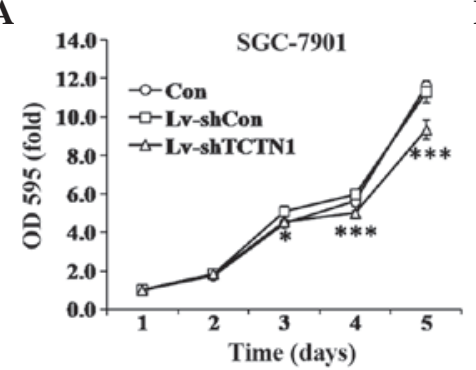

B

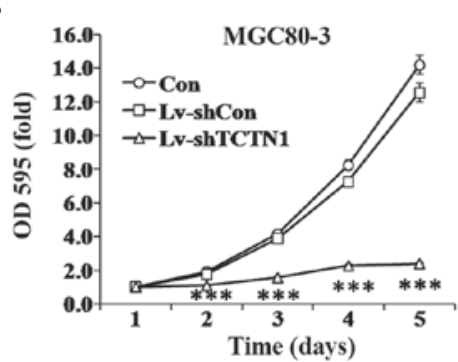

C

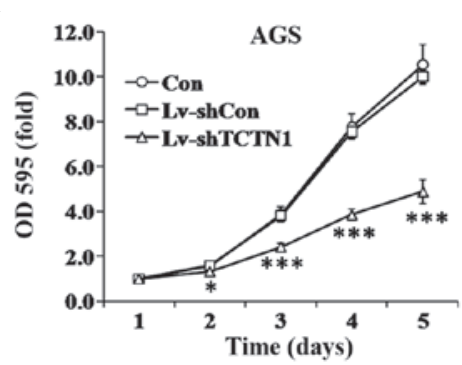

Figure 2. Proliferation of gastric cancer cells is attenuated following knockdown of TCTN1. Growth curves of (A) SGC-7901, (B) MGC80-3 and (C) AGS cells measured at day 1,2,3, 4 and 5 post infection by 3-(4, 5-dimethylthiazol-2-yl)-2, 5-diphenyltetrazolium bromide assay. " $\mathrm{P}<0.05$ and ${ }^{* * *} \mathrm{P}<0.001$, compared with Lv-shCon. TCTN1, tectonic 1 . 

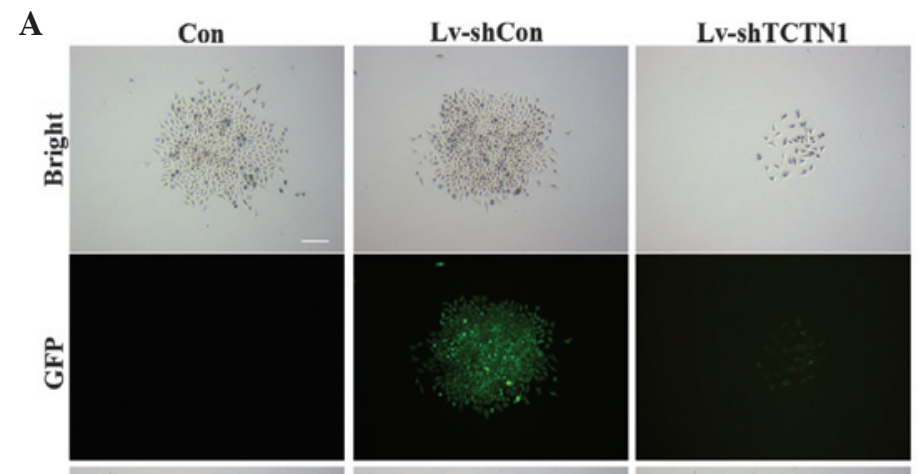

B
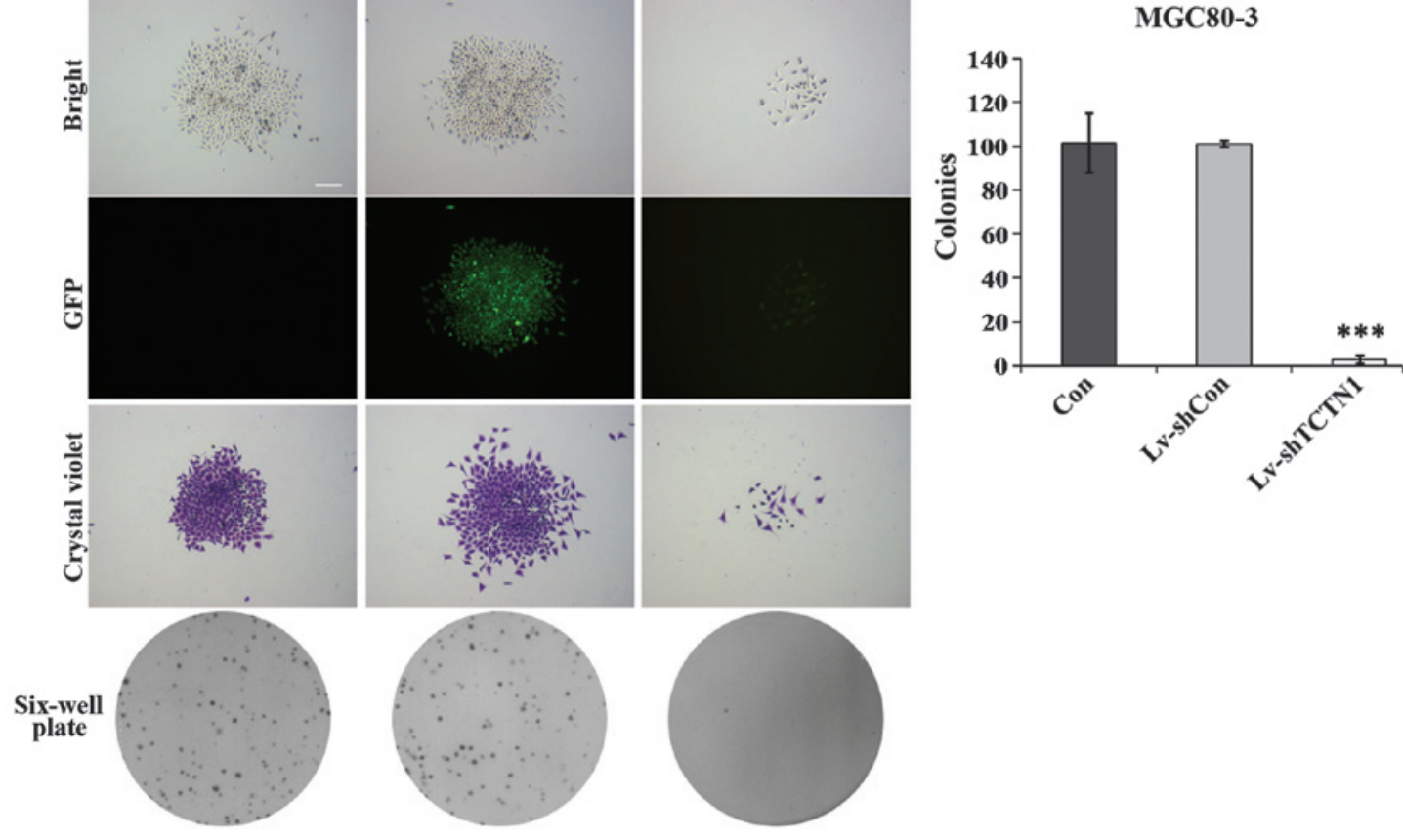

Figure 3. Colony formation of gastric cancer cells is decreased following knockdown of TCTN1. (A) Representative images of colonies formed in MGC80-3 cells infected with Lv-shCon or Lv-shTCTN1 (scale bar, $125 \mu \mathrm{m}$ ). Top, bright field; middle, GFP fluorescence; bottom, crystal violet staining. (B) Statistical calculation of colony numbers per well at day 7 post infection. ${ }^{* * *} \mathrm{P}<0.001$, compared with Lv-shCon. TCTN1, tectonic 1; GFP, green fluorescent protein.

$\mathbf{A}$
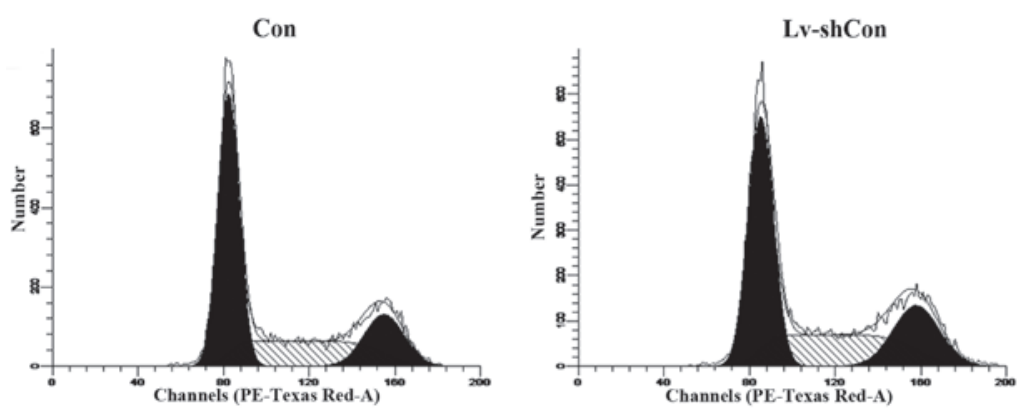

B
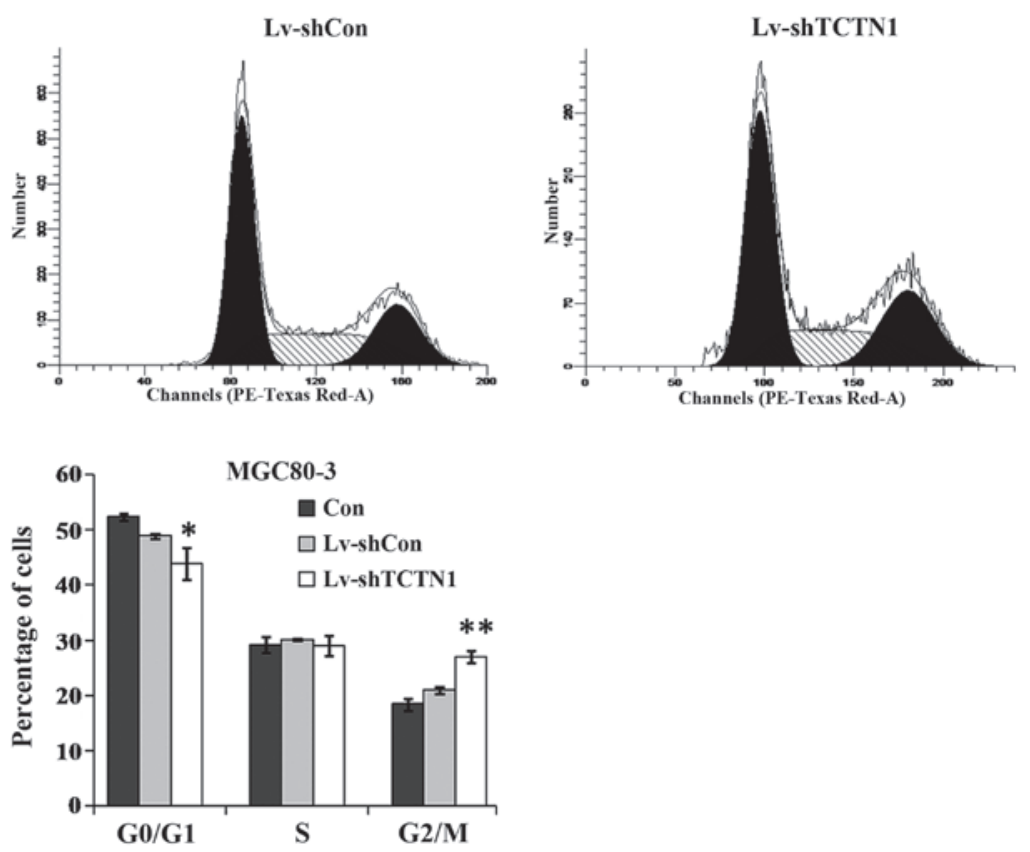

Figure 4. Cell cycle of MGC80-3 cells is blocked at G2/M phase following knockdown of TCTN1. (A) Representative graphs of the cell cycle distribution in MGC80-3 cells using propidium iodide staining by flow cytometry. (B) Statistical calculation of cell percentages in each cell cycle stage. ${ }^{*} \mathrm{P}<0.05$ and ${ }^{* *} \mathrm{P}<0.01$, compared with Lv-shCon. TCTN1, tectonic 1 .

\section{Results}

TCTN1 is suppressed efficiently in MGC80-3 cells by Lv-shTCTN1. The expression of TCTN1 was initially analyzed in different human gastric cancer cell lines by RT-qPCR. As shown in Fig. 1A, TCTN1 mRNA signals were detected in the three gastric cancer cell lines, SGC-7901, MGC80-3 and AGS.
Lentiviral vectors were then constructed expressing shTCTN1 and shCon, and recombinant lentivirus was obtained, Lv-shTCTN1 and Lv-shCon. As shown in Fig. 1B-D, the infection efficiency of recombinant lentivirus was detected in SGC-7901, MGC80-3 and AGS cells. More than $80 \%$ of cells presented GFP-positive signals, suggesting that recombinant lentivirus could deliver exogenous shRNA into gastric cancer 
cells with high efficiency. Furthermore, at day 5 post lentivirus infection, the mRNA levels of TCTN1 were significantly reduced in Lv-shTCTN1 groups, by 72.6, 63.2 and 95.8\% in SGC-7901, MGC80-3 and AGS, compared with the Lv-shCon groups (Fig. 1E-G). These results indicated that TCTN1 could be efficiently downregulated by the constructed Lv-shTCTN1.

Cell proliferation is inhibited following knockdown of TCTN1 in MGC80-3 cells. To investigate the function of TCTN1 in gastric cancer, the effect of TCTN1 knockdown on cell proliferation was examined by an MTT assay. As shown in Fig. 2A-C, the proliferation rates of Lv-shTCTN1 infected cells were significantly decreased, compared with Lv-shCon infected and uninfected cells. While Lv-shCon infected cells did not show significant difference compared with uninfected cells. The inhibition of proliferation was stronger in MGC80-3 and AGS cells $(\mathrm{P}<0.001)$ compared with SGC-7901 cells $(\mathrm{P}<0.05)$. These results suggested that TCTN1 may be essential for gastric cancer cell proliferation.

Colony formation is suppressed in MGC80-3 cells after TCTN1 knockdown. The colony forming ability of MGC80-3 cells was analyzed following knockdown of TCTN1 using crystal violet staining. As shown in Fig. 3A, the size of single colonies was smaller and the number of colonies formed was fewer in Lv-shTCTN1 groups than in control groups. The colony formation ability of LV-shTCTN1 infected cells was significantly decreased by $\sim 33$ fold compared with Lv-shCon infected and uninfected cells (Fig. 3B). These results indicated that TCTN1 knockdown could impair the colony formation ability of gastric cancer cells.

Cell cycle of MGC80-3 cells is blocked at $G_{2} / M$ phase following TCTN1 knockdown. To determine the mechanisms underlying impaired cell proliferation and colony formation, the cell cycle distribution of MGC80-3 cells was analyzed with PI staining using flow cytometry. As shown in Fig. 4, at day 3 post Lv-shTCTN1 infection, there were fewer cells in $\mathrm{G}_{0} / \mathrm{G}_{1}$ phase, while cell population in $\mathrm{G}_{2} / \mathrm{M}$ phase was significantly increased. The percentages of cells in $\mathrm{S}$ phase did not change significantly. The results suggested that downregulation of TCTN1 in MGC80-3 cells induced cell cycle arrest at $\mathrm{G}_{2} / \mathrm{M}$ phase.

\section{Discussion}

TCTN1, as a regulator of the Hh pathway, has been demonstrated to have an important role in regulating the Hh signaling pathway (20). The Hh pathway is important in development and cancer. Previous studies have demonstrated that inhibition of the Hh pathway using small interfering (si)RNA or chemical inhibitor could lead to growth suppression and apoptosis in several types of cancer, such as esophageal adenocarcinoma, ovarian cancer and endometrial cancer (25-27). Generally, siRNA targeting is effective over a short period of time, and gene transcription returns to normal after 3-7 days (28). However, lentivirus-based vectors have been used as a successful tool for gene targeting to deliver siRNA with long-term silencing efficiency (29). Therefore, in the present study, the biological role of TCTN1, a regulator of the Hh pathway, was examined via lentivirus-mediated siRNA in three gastric cancer cell lines.
Knockdown of TCTN1 markedly inhibited the proliferation and colony formation ability of gastric cancer cells, suggesting that TCTN1 is essential for gastric cancer growth.

Cell proliferation is directly linked to cell cycle progression. The cell cycle distribution of MGC80-3 cells was determined following lentivirus infection by flow cytometric analysis. The cell numbers in the $\mathrm{G}_{1}$ phase were decreased, and the cell population in $\mathrm{G}_{2} / \mathrm{M}$ phase was markedly increased in TCTN1 knockdown cells, indicating that downregulation of TCTN1 arrested cell cycle at $\mathrm{G}_{2} / \mathrm{M}$ phase. However, Chen et al (25) found that treatment of ovarian cancer with cyclopamine (inhibitor of $\mathrm{Hh}$ pathways) induced $\mathrm{G}_{1}$ arrest and apoptosis. The context differences between gastric cancer and ovarian cancer may also contribute to the results. Also, TCTN1 could have other functions in addition to regulating the Hh pathway. Recently, calcium signaling particularly Orail/ $\mathrm{Ca}^{2+}$ release-activated $\mathrm{Ca}^{2+}$ channel $\mathrm{M} 1$ has been shown to be the most important regulatory signal in cell cycle arrest as well as cancer cell proliferation $(30,31)$. Whether TCTN1 induced $\mathrm{G}_{2} / \mathrm{M}$ phase cell cycle arrest due to regulation of calcium signaling in gastric cancer remains to be investigated. Understanding the regulatory mechanism of TCTN1 in gastric cancer may provide a rational basis for drug development.

In conclusion, the present study demonstrated the novel biological functions of TCTN1 in human gastric cancer. Knockdown of TCTN1 suppressed the growth of gastric cancer cells through inducing $\mathrm{G}_{2} / \mathrm{M}$ phase arrest. The results suggest that TCTN1 may serve as a potential therapeutic target in human gastric cancer. However, the relative importance of TCTN1 in gastric carcinogenesis is not completely understood and warrants further investigation.

\section{Acknowledgements}

This study was supported by grants from the Natural Science Foundation of Zhejiang Province (grant no. Y205757), the Medical Research Fund of Zhejiang Province (grant no. 2009A029) and the Outstanding Research Personnel Training Funds of Zhejiang Cancer Hospital.

\section{References}

1. Wu WK, Cho CH, Lee CW, et al: Dysregulation of cellular signaling in gastric cancer. Cancer Lett 295: 144-153, 2010.

2. Danaei G, Vander Hoorn S, Lopez AD, Murray CJ and Ezzati M: Comparative risk assessment collaborating group (Cancers): Causes of cancer in the world: comparative risk assessment of nine behavioural and environmental risk factors. Lancet 366 : 1784-1793, 2005

3. Ramalho-Santos M, Melton DA and McMahon AP: Hedgehog signals regulate multiple aspects of gastrointestinal development. Development 127: 2763-2772, 2000.

4. Dessaud E, McMahon AP and Briscoe J: Pattern formation in the vertebrate neural tube: a sonic hedgehog morphogen-regulated transcriptional network. Development 135: 2489-2503, 2008.

5. Chiang C, Litingtung Y, Lee E, et al: Cyclopia and defective axial patterning in mice lacking Sonic hedgehog gene function. Nature 383: 407-413, 1996.

6. Yang L, Xie G, Fan Q and Xie J: Activation of the hedgehog-signaling pathway in human cancer and the clinical implications. Oncogene 29: 469-481, 2010.

7. Varjosalo M and Taipale J: Hedgehog: functions and mechanisms. Genes Dev 22: 2454-2472, 2008.

8. Saqui-Salces $M$ and Merchant JL: Hedgehog signaling and gastrointestinal cancer. Biochim Biophys Acta 1803: 786-795, 2010 . 
9. Kelleher FC: Hedgehog signaling and therapeutics in pancreatic cancer. Carcinogenesis 32: 445-451, 2011.

10. Shaw G, Price AM, Ktori E, et al: Hedgehog signalling in androgen independent prostate cancer. Eur Urol 54: 1333-1343, 2008.

11. Hui M, Cazet A, Nair R, Watkins DN, O'Toole SA and Swarbrick A: The Hedgehog signalling pathway in breast development, carcinogenesis and cancer therapy. Breast Cancer Res 15: 203, 2013.

12. Saran A: Basal cell carcinoma and the carcinogenic role of aberrant Hedgehog signaling. Future Oncol 6: 1003-1014, 2010.

13. Watkins DN, Berman DM and Baylin SB: Hedgehog signaling: progenitor phenotype in small-cell lung cancer. Cell Cycle 2: 196-198, 2003

14. Fu H, Hu Z, Wen J, Wang K and Liu Y: TGF-beta promotes invasion and metastasis of gastric cancer cells by increasing fascin1 expression via ERK and JNK signal pathways. Acta Biochim Biophys Sin (Shanghai) 41: 648-656, 2009.

15. Feng R, Xiao C and Zavros Y: The role of Sonic Hedgehog as a regulator of gastric function and differentiation. Vitam Horm 88 : 473-489, 2012

16. Wang XD, Inzunza $\mathrm{H}$, Chang $\mathrm{H}$, et al: Mutations in the hedgehog pathway genes SMO and PTCH1 in human gastric tumors. PLOS ONE 8: e54415, 2013.

17. Sun HJ, Liu YJ, Li N, et al: Sublocalization of Rab23, a mediator of Sonic hedgehog signaling pathway, in hepatocellular carcinoma cell lines. Mol Med Rep 6: 1276-1280, 2012.

18. Hou Q, Wu YH, Grabsch $\mathrm{H}$, et al: Integrative genomics identifies RAB23 as an invasion mediator gene in diffuse-type gastric cancer. Cancer Res 68: 4623-4630, 2008.

19. Katoh $Y$ and Katoh M: Hedgehog signaling pathway and gastrointestinal stem cell signaling network (review). Int J Mol Med 18: 1019-1023, 2006 (Review).

20. Reiter JF and Skarnes WC: Tectonic, a novel regulator of the Hedgehog pathway required for both activation and inhibition. Genes Dev 20: 22-27, 2006.
21. Garcia-Gonzalo FR, Corbit KC, Sirerol-Piquer MS, et al: A transition zone complex regulates mammalian ciliogenesis and ciliary membrane composition. Nat Genet 43: 776-784, 2011.

22. Alazami AM, Alshammari MJ, Salih MA, et al: Molecular characterization of Joubert syndrome in Saudi Arabia. Hum Mutat 33: 1423-1428, 2012.

23. Tiscornia G, Singer O and Verma IM: Production and purification of lentiviral vectors. Nat Protoc 1: 241-245, 2006.

24. Sakoda T, Kasahara N, Hamamori Y and Kedes L: A high-titer lentiviral production system mediates efficient transduction of differentiated cells including beating cardiac myocytes. J Mol Cell Cardiol 31: 2037-2047, 1999.

25. Chen X, Horiuchi A, Kikuchi N, et al: Hedgehog signal pathway is activated in ovarian carcinomas, correlating with cell proliferation: it's inhibition leads to growth suppression and apoptosis. Cancer Sci 98: 68-76, 2007.

26. Feng YZ, Shiozawa T, Miyamoto T, et al: Overexpression of hedgehog signaling molecules and its involvement in the proliferation of endometrial carcinoma cells. Clin Cancer Res 13: 1389-1398, 2007.

27. Zaidi AH, Komatsu Y, Kelly LA, et al: Smoothened inhibition leads to decreased proliferation and induces apoptosis in esophageal adenocarcinoma cells. Cancer Invest 31: 480-489, 2013.

28. McManus MT SP: Gene silencing in mammals by small interfering RNAs. Nat Rev Genet 3: 737-747, 2002.

29. Devi G: siRNA-based approaches in cancer therapy. Cancer Gene Ther 13: 819-829, 2006

30. Hou MF, Kuo HC, Li JH, et al: Orai1/CRACM1 overexpression suppresses cell proliferation via attenuation of the store-operated calcium influx-mediated signalling pathway in A549 lung cancer cells. Biochim Biophys Acta 1810: 1278-1284, 2011.

31. Yang IH, Tsai YT, Chiu SJ, et al: Involvement of STIM1 and Orail in EGF-mediated cell growth in retinal pigment epithelial cells. J Biomed Sci 20: 41, 2013. 